\title{
A New Representation of the k-Gamma Functions
}

\author{
Asifa Tassaddiq \\ College of Computer and Information Sciences, Majmaah University, Majmaah 11952, Saudi Arabia; \\ a.tassaddiq@mu.edu.sa
}

Received: 8 December 2018; Accepted: 19 January 2019; Published: 1 February 2019

\begin{abstract}
The products of the form $z(z+l)(z+2 l) \ldots(z+(k-1) l)$ are of interest for a wide-ranging audience. In particular, they frequently arise in diverse situations, such as computation of Feynman integrals, combinatory of creation, annihilation operators and in fractional calculus. These expressions can be successfully applied for stated applications by using a mathematical notion of k-gamma functions. In this paper, we develop a new series representation of k-gamma functions in terms of delta functions. It led to a novel extension of the applicability of k-gamma functions that introduced them as distributions defined for a specific set of functions.
\end{abstract}

Keywords: series representation; gamma function; k-gamma function; k-Pochhammer symbol; delta functions; Fourier transform; test functions; distributions

\section{Introduction}

Diaz and Pariguan [1] introduced and investigated k-gamma functions when they were evaluating Feynman integrals. These integrals are of fundamental significance in high-energy physics because they offer a general integral representation of the involved functions [2]. Since then, k-gamma functions have emerged with diverse effects on mathematics and applications. Karwowski and Witek [3] have used such functions while solving the Schrodinger equation for harmonium and related models in view of important applications in quantum chemistry. The measure-theoretic and combinatorial versions for the k-increasing factorial numbers that appear as an illustration and the combinatorics of the Pochhammer k-symbol has appealed extensively in the collected works. For example, Lackner and Lackner [4] have used k-gamma functions for combinatorial analysis in view of important applications in statistics. Such applications of different forms of k-gamma functions have put away the prominent concern, and several papers have subsequently been offered involving the analysis of k-gamma functions. For modeling real-life problems, the role of fractional calculus [5] is vibrant. It is remarkable that k-gamma functions and related k-Pochhammer symbols are more the functions of the fractional calculus. For example, Agarwal et al [6] have solved fractional kinetic equations comprising of k-Mittag-Leffler functions. Set et al. have used the analogue to the Riemann-Liouville singular kernel at k-calculus in [7]. For more comprehensive and detailed studies of related works, I refer the interested reader to [1-14] and associated references therein. The literature review for k-gamma functions motivates us to state "on the one hand k-gamma functions excited to mathematician for the analysis of mathematical concepts in a new way and on the other applications of these functions in diverse problems are fundamental".

By taking inspiration from the above discussion, in our current investigation, we acquire a new representation for the k-gamma functions in terms of complex delta functions by following the methodology of Chaudhry \& Qadir [15], Tassaddiq \& Qadir [16,17], Tassaddiq [18], Lail \& Qadir [19], Tassaddiq et al [20] and Tassaddiq [21,22]. In these articles [15-22], the authors have investigated new representations for gamma, generalized gamma, Hypergeometric functions, extended Fermi-Dirac and Bose-Einstein functions and $\lambda$-generalized Hurwitz-Lerch zeta functions, respectively. Such 
investigations are significant for providing considerable and inventive identities for such higher transcendental functions. It offers an analytical skill to evaluate different integrals that are not found in the literature see for example [23,24].

To comprehend the results of this paper, some fundamental mathematical notions about k-gamma and test function space are outlined in Section 2. These are stated in Sections 2.1 and 2.2, respectively. The remaining paper is organized as: Our main results about k-gamma functions in the form of a series of delta functions are given in Section 3.1. A discussion about the behavior and applications of the new series is provided in Section 3.2. The cogency of the results is discussed in Section 3.4. This investigation is concluded in Section 4 by highlighting the future directions about the further new results.

\section{Materials and Methods}

\section{1. $k$-Gamma Functions}

Thinking through the usual notations,

$$
N:=\{1,2,\} ; N_{0}:=N \cup\{0\} ; Z^{-}:=\{-1,-2,\} ; Z_{0}^{-}:=Z^{-} \cup\{0\},
$$

The set of negative integers is denoted by $Z^{-}$, the set of real is denoted by $R$, positive real by $R^{+}$, and complex numbers by $C$ in this article.

The extension of factorial from natural to complex numbers in the form of gamma function was investigated by Euler (1707-1783). Now, it is a commonly used function and can be represented in the form of integral [25] as follows:

$$
\Gamma(z)=\int_{0}^{\infty} t^{z-1} e^{-t} d t ; \Re(z)>0 .
$$

The focus point of this paper, namely k-gamma function, is an extension for the Euler gamma function:

$$
\Gamma_{k}(z)=\int_{0}^{\infty} t^{z-1} e^{\frac{-t^{k}}{k}} d t(k \in R),
$$

which is introduced and defined by Diaz and Pariguan [1] in 2007. For $k=1$, it reduces to original gamma function $\Gamma_{1}(z)=\Gamma(z)$ and for $k=2$, it reduces to an integral of Gaussian functions:

$$
\Gamma_{2}(z)=\int_{0}^{\infty} t^{z-1} e^{\frac{-t^{2}}{2}} d t
$$

That appears in many applications [12]. By making a substitution $u=\frac{t^{k}}{k}$ in the above Equation (3), we can find the relation $\Gamma_{k}(z)=k^{\frac{z}{k-1}} \Gamma\left(\frac{z}{k}\right)$ and all the known properties of gamma functions can be generalized for k-gamma functions.

The shifted factorial $(\Lambda)_{\rho}$ namely Pochhammer symbols are defined in the relationship of the elementary gamma function and are given as:

$$
(\Lambda)_{\rho}=\frac{\Gamma(\Lambda+\rho)}{\Gamma(\Lambda)}=\left\{\begin{array}{c}
1(\rho=0, \Lambda \in C) \\
\Lambda(\Lambda+1) \ldots(\Lambda+k-1)(\rho=k \in \mathbb{N} ; \Lambda \in \mathbb{C} \backslash\{0\}),
\end{array}\right.
$$

The commonly occurring products $z(z+l)(z+2 l) \ldots(z+(k-1) l)$ naturally provide k-Pochammar symbols $(\alpha)_{\kappa, n}$ defined as:

$$
(\Lambda)_{\kappa, \rho}=\frac{\Gamma_{k}(\Lambda+\kappa \rho)}{\Gamma_{k}(\Lambda)} .
$$


Several authors studied the properties and applications of these k-gamma functions. It also led to several new results for gamma functions. Some of these are stated here [1]:

$$
\left.\begin{array}{c}
\Gamma_{k}(k)=1 \\
(z)_{n, k}=\frac{\Gamma_{k}(z+n k)}{\Gamma_{k}(z)} \\
\Gamma_{k}(z+k)=z \Gamma_{k}(z) \\
\Gamma_{k}(z+k)=z \Gamma_{k}(z) \\
\Gamma_{k}(z) \Gamma_{k}(k-z)=\frac{\pi}{\sin \frac{\pi z}{k}} \\
\Gamma_{k}\left(\frac{x}{p}+\frac{y}{q}\right) \leq\left(\Gamma_{k}(x)\right)^{\frac{1}{p}}\left(\Gamma_{k}(x)\right)^{\frac{1}{q}} x, y \in R ; 1<p<\infty ; \frac{1}{p}+\frac{1}{q}=1
\end{array}\right\}
$$

For more such properties of k-gamma and related functions, we refer the reader to [1-14] and related bibliography therein. The subsequent Section 2.2 is taken from author work [22].

\subsection{Distributions and Test Functions}

Generalized functions (or distributions) are the components of the dual space of test functions space. The appraisal of these functions is substantial since they also comprise of singular functions. So, they can be defined, differentiated and integrated similar to functions. A famous example of a singular distribution is a delta function that satisfy:

$$
\langle\delta(z-\omega), \Lambda(z)\rangle=\Lambda(\omega)(\forall \Lambda \in D, \omega \in R),
$$

And for a non-zero $\omega, \delta(-z)=\delta(z) ; \delta(\omega z)=\frac{\delta(z)}{|\omega|}$.

Gelfand and Shilov [26] delivered an abundant exposition for generalized functions. The frequently used spaces of test functions are the spaces of compact support functions denoted by $D$, and the space of rapidly decaying functions denoted by $S$, that also have derivatives of all orders. The spaces $D^{\prime}$ and $S^{\prime}$ are the dual spaces of $D$ and $S$. Spaces $S$ and $S^{\prime}$ are closed under the Fourier transform, but $D$ and $D^{\prime}$ are not. The Fourier transform of the elements of $D^{\prime}$ are continuous linear functionals acting on the elements of $Z$ that comprise of entire functions such that their Fourier transforms are in $D$ [27]. The entire function $\Lambda \in Z$ does not vanish on some interval $\omega_{1}<z<\omega_{2}$, but vanishes universally. Accordingly:

$$
Z^{\prime} \supset S^{\prime} \supset S \supset Z ; D \cap Z \equiv 0 ; D^{\prime} \supset S^{\prime} \supset S \supset D .
$$

The elements of $Z$ consist of entire analytic functions satisfying the following set of inequalities:

$$
\left|z^{p} \Lambda(z)\right| \leq C_{p} e^{\eta|\theta|} ;(p=0,1,2, \ldots)
$$

where the constants $\eta$ and $C_{p}$ may depend on $\Lambda$. The Fourier transform of exponential function is

$$
F\left[e^{\alpha t} ; \theta\right]=2 \pi \delta(\theta-i \alpha)
$$

As an example of distribution that is an element of $Z^{\prime}$ and for $\forall g \in Z^{\prime}$

$$
g(z+b)=\sum_{i=0}^{\infty} g^{(i)}(z) \frac{b^{i}}{i !} .
$$

So that we have the following basic identity:

$$
\begin{gathered}
\delta(z+b)=\sum_{i=0}^{\infty} \delta^{(i)}(z) \frac{b^{i}}{i !} ; \delta^{(i)}(z), \Lambda(z)=(-1)^{r} \Lambda^{(i)}(0) . \\
\delta\left(\omega_{1}-z\right) \delta\left(z-\omega_{2}\right)=\delta\left(\omega_{1}-\omega_{2}\right) .
\end{gathered}
$$


The elementary functions like Sine, Cosine, Hyperbolic Sine and Cosine are some examples of distributions in $D^{\prime}$ whose Fourier transforms being delta function are in $Z^{\prime}$. For an additional extensive study of these spaces, we refer the reader to [26-29] and the related bibliography therein.

During this research, conditions on the parameters will be considered standard as given in this section unless otherwise stated.

\section{Results}

\subsection{New Series Representation of the $k$-Gamma Functions}

In mathematics, generalized functions or distributions are objects extending the notion of functions. These are the general form of the conventional function. The k-gamma function is a conventional function. For which we find a series of delta functions. The delta functions are generalized functions that are meaningful only when defined as an inner product with a suitable function (as discussed in detail in Section 2.2). In this section, we will compute our results using conventional methods but how they can be applied rigorously in the sense of distributions will be discussed in the next section.

Theorem 1. The k-gamma functions have the following representation.

$$
\Gamma_{k}(z)=2 \pi \sum_{n=0}^{\infty} \frac{\left(-\frac{1}{k}\right)^{n}}{n !} \delta(\theta-i(v+n k)) .
$$

Proof. Let us first replace $t=e^{x}$ and $z=v+i \theta$ in Equation (3) then we get:

$$
\Gamma_{k}(z)=\int_{-\infty}^{\infty} e^{x(v+i \theta)} \exp \left(-\frac{e^{k x}}{k}\right) d x, \quad(k \in R) .
$$

Now writing the series form of the exponential function:

$$
\exp \left(-\frac{e^{k x}}{k}\right)=\sum_{n=0}^{\infty} \frac{\left(-\frac{e^{k x}}{k}\right)^{n}}{n !}
$$

And then collecting and expanding the exponential terms:

$$
\Gamma_{k}(z)=\int_{-\infty}^{\infty} e^{x(v+i \theta)} \sum_{n=0}^{\infty} \frac{\left(-\frac{e^{k x}}{k}\right)^{n}}{n !} d x, \quad(k \in R),
$$

We get:

$$
\Gamma_{k}(z)=\sum_{n=0}^{\infty} \frac{\left(-\frac{1}{k}\right)^{n}}{n !} \int_{-\infty}^{\infty} e^{i x \theta} e^{(v+n k) x} d x .
$$

We can interchange the operations of integration and summation because of uniform convergence of the integral. By means of Equation (11), we find:

$$
\int_{-\infty}^{\infty} e^{i \theta x} e^{(v+n k) x} d x=F\left[e^{(v+n k) x} ; \theta\right]=2 \pi \delta(\theta-i(v+n k))
$$

These Equalities (19)-(20) combined together produces the result (15) as mentioned above.

Remark 1. One can develop equivalent outcomes for supplementary related functions, in the form of following corollaries. 
Corollary 1. k-Gamma functions have the subsequent demonstration:

$$
\Gamma_{k}(z)=2 \pi \sum_{n, r=0}^{\infty} \frac{\left(-\frac{1}{k}\right)^{n}}{n !} \frac{(-i(v+n k))^{r}}{r !} \delta^{(r)}(\theta) .
$$

Proof. This is achieved by considering and combining Equations (13) and (15) as follows:

$$
\delta(\theta-i(v+n k))=\sum_{r=0}^{\infty} \delta^{(r)}(z) \frac{(-i(v+n k))^{r}}{r !} ; \delta^{(r)}(z), \Lambda(z)=(-1)^{r} \Lambda^{(r)}(0)
$$

Now using this modified form in (15), we get the required result.

Corollary 2. k-Gamma functions have the subsequent demonstration:

$$
\Gamma_{k}(z)=2 \pi \sum_{n=0}^{\infty} \frac{\left(-\frac{1}{k}\right)^{n}}{n !} \delta(z+n k) .
$$

Proof. This follows by a simple modification of (20) as follows:

$$
\begin{aligned}
& \int_{-\infty}^{\infty} e^{i x \theta} e^{(v+n k) x} d x=F {\left[e^{(v+n k) x} ; \theta\right]=2 \pi \delta(\theta-i(v+n k))=2 \pi \delta\left[\frac{1}{i}(i \theta+(v+n k))\right] } \\
&=2 \pi|i| \delta(v+i \theta+n k)=2 \pi \delta(z+n k) .
\end{aligned}
$$

Now using this modified form in (15), we get the required result.

Corollary 3. k-Gamma functions have the subsequent demonstration:

$$
\Gamma_{k}(z)=2 \pi \sum_{n, r=0}^{\infty} \frac{\left(-\frac{1}{k}\right)^{n}}{n !} \frac{(n k)^{r}}{r !} \delta^{(r)}(z) .
$$

Proof. This is achieved by considering Equation (13).

$$
\delta(z+n k)=\sum_{r=0}^{\infty} \frac{(n k)^{r}}{r !} \delta^{(r)}(z) ;\left\langle\delta^{(r)}(z), \Lambda(z)\right\rangle=(-1)^{r} \Lambda^{(r)}(0) .
$$

and combining the result with (23).

Corollary 4. Gamma functions have the following representation ([1], p. 2092, Equation (2.7))

$$
\Gamma(z)=\Gamma_{1}(z)=2 \pi \sum_{n=0}^{\infty} \frac{(-1)^{n}}{n !} \delta(\theta-i(v+n)) .
$$

Proof. The result follows simply by replacing $k=1$ in Equation (15).

Corollary 5. Gamma functions have the following representation:

$$
\Gamma(z)=2 \pi \sum_{n, r=0}^{\infty} \frac{(-1)^{n}}{n !} \frac{(-i(v+n))^{r}}{r !} \delta^{(r)}(\theta) .
$$

Proof. The result follows simply by replacing $k=1$ in Equation (21). 
Corollary 6. Gamma functions have the following representation:

$$
\Gamma(z)=2 \pi \sum_{n=0}^{\infty} \frac{(-1)^{n}}{n !} \delta(z+n) .
$$

Proof. The result follows simply by replacing $k=1$ in Equation (23).

Corollary 7. Gamma functions have the following representation:

$$
\Gamma(z)=2 \pi \sum_{n, r=0}^{\infty} \frac{(-1)^{n}}{n !} \frac{n^{r}}{r !} \delta^{(r)}(z) .
$$

Proof. The result follows simply by replacing $k=1$ in Equation (25).

Note that we have acquired a series in terms of Dirac delta functions that are only significant in the sense of distributions once defined as an inner product with some appropriate function. For a simple observation, multiply Equation (29) with $\frac{1}{\Gamma(z)}$ in a conventional way.

$$
1=\frac{2 \pi \sum_{n=0}^{\infty} \frac{(-1)^{n}}{n !} \delta(z+n)}{\Gamma(z)}
$$

Note that, at $z=-n$, the singularities of delta function are canceled by the zeros of the reciprocal gamma function in the involved product $\lim _{z \rightarrow-n} \frac{\delta(z+n)}{\Gamma(z)}=\lim _{z \rightarrow-n} \frac{\frac{1}{z+n}}{\frac{1}{z+n}}=\lim _{z \rightarrow-n} \frac{z+n}{z+n}=1$. Therefore, by using the basic definition of delta function:

$$
\delta(t)=\left\{\begin{array}{cc}
\infty & (t=0) \\
0 & (t \neq 0)
\end{array}\right.
$$

In Equation (31), we obtain:

$$
1= \begin{cases}\frac{2 \pi}{e} ; & z=-n \\ 0 ; & z \in \mathbb{C} \backslash\{-n\}\end{cases}
$$

That is an apparent inconsistency.

For the moment, if we think through the inner product

$$
\left\langle\Gamma(z), \frac{1}{\Gamma(z)}\right\rangle=2 \pi \sum_{n=0}^{\infty} \frac{(-1)^{n}}{n !}\left\langle\delta(z+n), \frac{1}{\Gamma(z)}\right\rangle
$$

We get

$$
\int_{z \in \mathbb{C}} 1 d z=2 \pi \sum_{n=0}^{\infty} \frac{(-1)^{n}}{n ! \Gamma(-n)}
$$

Since $\frac{1}{\Gamma(-n)}=0, n=0,1,2, \ldots$ so we get from Equation (35)

$$
\begin{gathered}
\int_{z \in \mathbb{C}} 1 d z=0 \\
\int_{z \in \mathbb{C}} 1 d z=\int_{-\infty}^{+\infty} 1 d z=0 \\
\Rightarrow \infty=0
\end{gathered}
$$

And again, an apparent inconsistency. So, we should be precise in choosing a space of functions to consider the convergence of this series representation. 


\subsection{Convergence and Applications of New Representation}

The representation of the $k$-gamma functions $\Gamma_{k}(z)$ is achieved as an infinite sum of delta functions that is definite if it converges in the distributional sense. Hence, it is interesting to prove that a series of delta functions are distributions (generalized functions) on the space $Z$ as illustrated in the following theorem.

Theorem 2. Show that k-gamma function is defined as a distribution for space Z.

Proof. Consider the following combination for arbitrary $\Lambda_{1}(z), \Lambda_{2}(z) \in Z$ and $c_{1}, c_{2} \in C$.

$$
\begin{gathered}
\left\langle\Gamma_{k}(z), c_{1} \Lambda_{1}(z)+c_{2} \Lambda_{2}(z)\right\rangle=\left\langle 2 \pi \sum_{n=0}^{\infty} \frac{\left(-\frac{1}{k}\right)^{n}}{n !} \delta(z+k n), c_{1} \Lambda_{1}(z)+c_{2} \Lambda_{2}(z)\right\rangle . \\
\Rightarrow\left\langle\Gamma_{k}(z), c_{1} \Lambda_{1}(z)+c_{2} \Lambda_{2}(z)\right\rangle=c_{1}\left\langle\Gamma_{k}(z), \Lambda_{1}(z)\right\rangle+c_{2}\left\langle\Gamma_{k}(z), \Lambda_{2}(z)\right\rangle .
\end{gathered}
$$

Next, consider any sequence $Z \supset\left\{\Lambda_{\mu}\right\}_{\mu=1}^{\infty} \rightarrow 0$ and the fact that $\delta(z)$ is continuous over $Z$ we can assert that $\left\{\left\langle\delta(z+k n), \Lambda_{\mu}\right\rangle\right\}_{\mu=1}^{\infty} \rightarrow 0$.

$$
\Rightarrow\left\{\left\langle\Gamma_{k}(z), \Lambda_{\mu}(z)\right\rangle\right\}_{\mu=1}^{\infty}=2 \pi \sum_{n=0}^{\infty} \frac{\left(-\frac{1}{k}\right)^{n}}{n !}\left\{\left\langle\delta(z+k n), \Lambda_{\mu}(z)\right\rangle\right\}_{\mu=1}^{\infty}
$$

converges to zero. Hence, k-gamma function is a generalized function over $Z$ in view of the convergence of series representation (15) as follows:

$$
\begin{aligned}
\left\langle\Gamma_{k}(z), \Lambda(z)\right\rangle= & 2 \pi \sum_{n=0}^{\infty} \frac{\left(-\frac{1}{k}\right)^{n}}{n !}\langle\delta(z+n k), \Lambda(z)\rangle ;(\forall \Lambda(z) \in) \\
& =2 \pi \sum_{n=0}^{\infty} \frac{\left(-\frac{1}{k}\right)^{n}}{n !} \Lambda(-n k),
\end{aligned}
$$

where we have used the shifting property of delta functions:

$$
\langle\delta(z+n k), \Lambda(z)\rangle=\Lambda(-n k),
$$

which being the elements of space $Z$ are slowly increasing (bounded by a polynomial) test functions and note that sum over the coefficients is:

$$
\begin{aligned}
& \text { sum over the coefficients }=\sum_{n=0}^{\infty} \frac{\left(-\frac{1}{k}\right)^{n}}{n !} \\
& =\exp \left(-\frac{1}{k}\right) .
\end{aligned}
$$

This sum is finite and well defined. So from Equation (42), it is evident that $\left\langle\Gamma_{k}(z), \Lambda(z)\right\rangle$ being a product of a rapidly decreasing and slowly increasing function is convergent, for $\forall \Lambda(z) \in$. It can also be verified in view of well-known Abel theorem to check the behavior of series. It indicates the same statement for other specific cases as presented in Equations (21)-(29).

In this investigation, the convergence is shown for the functions of slow growth; nevertheless, it can be perceived that the series converges for a wider space of functions. Therefore, for $\forall \Lambda(z) \in$ the investigated sum is finite, but if this sum is finite, then $\Lambda(z)$ may be an element of some wider domain where delta function is defined. 
Subsequently, by means of this acquired representation of $\Gamma_{k}(z)$ we establish some integral identities and validate these identities by making use of standard Fourier transform in the ensuing section. Initially, we thought through a simple example of particular test functions

$$
\Lambda(z)=\tau^{z \xi}(\xi>0 ; z \in C)
$$

Given this Equation (15) and shifting characteristic of delta functions, the product $\left\langle\Lambda(z), \Gamma_{k}(z)\right\rangle$ is given by the following identity:

$$
\int_{z \in \mathbb{C}} \tau^{z \xi} \Gamma_{k}(z) d z=2 \pi \sum_{n=0}^{\infty} \frac{\left(-\frac{1}{k}\right)^{n}}{n !} \tau^{-n k \xi}=2 \pi \sum_{n=0}^{\infty} \frac{\left(-\frac{\tau^{-k \xi}}{k}\right)^{n}}{n !}=2 \pi \exp \left(-\frac{\tau^{-k \xi}}{k}\right)
$$

Likewise, in view of the acquired representations (21)-(29), we get the following new results as a particular case of (45) with $\mathrm{k}=1$ :

$$
\int_{z \in \mathbb{C}} \tau^{z \xi} \Gamma(z) \mathrm{d} z=2 \pi \sum_{n=0}^{\infty} \frac{(-1)^{n}}{n !} \tau^{-n \xi}=2 \pi \sum_{n=0}^{\infty} \frac{\left(-\tau^{-\xi}\right)^{n}}{n !}=2 \pi \exp \left(-\tau^{-\xi}\right)
$$

Remark 2. Series of delta functions also occur as an electromotive force in different engineering problems. It can be noted that any sequence of delta functions $\{\delta(s+n k)\}_{n=0}^{\infty}$ multiplied by $2 \pi \exp \left(-\frac{1}{k}\right)$ results in $k$-gamma functions and for $k=1$, similar result is true for gamma functions.

Remark 3. The gamma functions has a classical representation ([30], p. 2)

$$
\Gamma(z)=2 \pi \sum_{n=0}^{\infty} \frac{(-1)^{n}}{n !} \frac{1}{z+n}
$$

which demonstrates that gamma function has poles at negative integers $z=-n ; n=0,1,2,3, \ldots$ and the sum over the residues of these poles is $\sum_{n=0}^{\infty} \frac{(-1)^{n}}{n !}$. Now if we compare this representation with (29), then the result can be interpreted in the same way.

Remark 4. Similarly, the k-gamma functions has a classical representation:

$$
\Gamma_{k}(z)=2 \pi \sum_{n=0}^{\infty} \frac{\left(-\frac{1}{k}\right)^{n}}{n !} \frac{1}{z+n k}
$$

which demonstrates that $k$-gamma function has poles at negative integers $z=-n k ; n k=0,1,2,3, \ldots$ and sum over the residues of these poles is $\sum_{n=0}^{\infty} \frac{\left(-\frac{1}{k}\right)^{n}}{n !}$. Now if we compare this representation with our new representation (23), then the result can be interpreted in the same way.

These outcomes provide additional understanding of more results, for example specifying $\tau=e^{-1}$ in Equation (45), we get Laplace transform of $\Gamma_{k}(z)$. Formerly, we need to take account of the stability of these results as a part of the next section.

\subsection{Verification of the Results Obtained by New Representation}

Central inspiration for this part is to validate the stability of the results achieved by new demonstration with the standard Fourier transform.

By specifying $t=e^{x}$ and $z=v+i \xi$ in Equation (3), the $k$-gamma functions have a Fourier transform representation as follows:

$$
\Gamma_{k}(v+i \theta)=\sqrt{2 \pi} F\left[e^{v x} \exp \left(-\frac{e^{k x}}{k}\right) ; \xi\right](k \in R),
$$


And considering $k=1$, we develop:

$$
\Gamma(v+i \theta)=\sqrt{2 \pi} F\left[e^{v x} \exp \left(-e^{x}\right) ; \theta\right]
$$

The dual characteristics of the Fourier transform of any function $u(t)$, is given as

$$
F[\sqrt{2 \pi} F[u(t) ; \theta] ; \xi]=2 \pi u(-\xi)
$$

Therefore, an application of this characteristic to the above Equations (49)-(50), leads to the following result:

$$
F\left\{\Gamma_{k}(v+i \theta) ; \xi\right\}=F\left[\sqrt{2 \pi} F\left[e^{v x} \exp \left(-\frac{e^{k x}}{k}\right)\right] ; \xi\right]=f(-\xi)=2 \pi e^{-v \xi} \exp \left(-\frac{e^{k \xi}}{k}\right),
$$

Equivalently,

$$
\int_{-\infty}^{+\infty} e^{i \theta \xi} \Gamma_{k}(\nu+i \theta) d \theta=2 \pi e^{-v \xi} \exp \left(-\frac{e^{k \xi}}{k}\right),
$$

That can be obtained as a particular case of our key result (45) by specifying $\tau=e ; z=v+i \theta$. This discussion illustrates that the consequences of our new demonstration are reliable with the well-established results. Moreover, by specifying $\xi=0$ in this identity namely (52), we develop the subsequent expression:

$$
\int_{-\infty}^{+\infty} \Gamma_{k}(v+i \theta) d \theta=2 \pi \exp \left(-\frac{1}{k}\right)
$$

That can be obtained as a particular case of our key result (45). It confirms that our general representation yield new results that cannot be achieved by existing procedures nevertheless particular cases of these new results are reliable with the results obtained by existing methods. Similarly, by considering $k=1$ in the above equations, we can get the following integrals of gamma functions. We can observe that identities found in our investigation are novel and innovative while preserving the consistency for special cases obtained by well-studied Fourier transform methods.

$$
\begin{gathered}
\int_{-\infty}^{+\infty} e^{i \theta \xi} \Gamma_{1}(v+i \theta) d \theta=\int_{-\infty}^{+\infty} e^{i \theta \xi} \Gamma(\nu+i \theta) d \theta=\frac{2 \pi e^{-v \xi}}{e}, \\
\int_{-\infty}^{+\infty} \Gamma(\nu+i \theta) d \theta=\frac{2 \pi}{e} .
\end{gathered}
$$

To approve the stability of these new identities, one can observe that the identities (52)-(55) can be produced as particular forms of the Equation (45) for $\tau=e, z=v+i \theta$ and vice versa. These results (54-55) are direct to achieve by means of a simple element of Fourier transform and consequently, at this point, to check the solidity of new demonstration it comes to be more significant.

Remark 5. In light of remark 3 and 4, it can be observed that all of the integrals obtained in this investigation only contribute some of the residues for the involved singularities of the corresponding integrand. It also verifies the important result of the integration theory.

By means of Parseval's characteristics of Fourier transform for the representation given by Equation (49), one can apply the following integral formulae:

For k-gamma functions $\Gamma_{k}(z)$,

$$
\int_{-\infty}^{+\infty} \Gamma_{k}(\nu+i \theta) \overline{\Gamma_{k}(\mu+i \theta)} d \theta=2 \pi \int_{0}^{\infty} t^{v+\mu-1} e^{-\frac{2 t^{k}}{k}} d t=\pi 2^{1-\frac{(v+\mu)}{k}} \Gamma_{k}(\nu+\mu)
$$

And $k=1$ leads to the following known result ([16], p. 2092, Equation (3.2))

$$
\int_{-\infty}^{+\infty} \Gamma(v+i \theta) \overline{\Gamma(\mu+i \theta)} d \theta=2 \pi \int_{0}^{\infty} t^{v+\mu-1} e^{-2 t} d t=\pi 2^{1-v-\mu} \Gamma(\nu+\mu) .
$$


Taking $v=\mu$, we get:

$$
\begin{gathered}
\int_{-\infty}^{+\infty}\left|\Gamma_{k}(v+i \theta)\right|^{2} d \tau=2 \pi \int_{0}^{\infty} t^{2 v-1} e^{-\frac{2 t^{k}}{k}} d t=\pi 2^{1-\frac{2 v}{k}} \Gamma_{k}(2 v), \\
\int_{-\infty}^{+\infty}|\Gamma(v+i \theta)|^{2} d \tau=\int_{0}^{\infty} t^{2 v-1} e^{-2 t} d t=\pi 2^{1-2 v} \Gamma(2 v) .
\end{gathered}
$$

\subsection{Further Distributional Properties of k-Gamma Function}

Taking inspiration from ([[27]], Chapter 7), here we enlist some properties of k-gamma functions in the form of a theorem.

Theorem 3. For an arbitrary test function $\Lambda(z) \in Z$, k-gamma functions do satisfy the following charactristics in the sense of distributions:

1. $\left\langle\Gamma_{k}(z), \Lambda_{1}(z)+\Lambda_{2}(z)\right\rangle=\left\langle\Gamma_{k}(z), \Lambda_{1}(z)\right\rangle+\left\langle\Gamma_{k}(z), \Lambda_{2}(z)\right\rangle$,

2. $\left\langle c_{1} \Gamma_{k}(z), \Lambda(z)\right\rangle=\left\langle\Gamma_{k}(z), c_{1} \Lambda(z)\right\rangle$,

3. $\left\langle\Gamma_{k}(z-\Gamma), \Lambda(s)\right\rangle=\left\langle\Gamma_{k}(s), \Lambda(s+\Gamma)\right\rangle$,

4. $\left\langle\Gamma_{k}\left(c_{1} z\right), \Lambda(z)\right\rangle=\left\langle\Gamma_{k}(z), \frac{1}{c_{1}} \Lambda\left(\frac{z}{c_{1}}\right)\right\rangle$,

5. $\left\langle\Gamma_{k}\left(c_{1} z-\Gamma\right), \Lambda(z)\right\rangle=\left\langle\Gamma_{k}(z), \frac{1}{c_{1}} \Lambda\left(\frac{z}{c_{1}}+\Gamma\right)\right\rangle$

6. For a regular distrbution $\psi(z), \psi(z) \Gamma_{k}(z) \in Z$ is a continuous linear functional over $Z$.

7. $\Gamma_{k}(z+1)=z \Gamma_{k}(z)$ iff $\Lambda(z-1)=z \Lambda(z)$ where $\Lambda \in Z$

8. $\left\langle\Gamma_{k}^{(m)}(z), \Lambda(z)\right\rangle=\sum_{n=0}^{\infty} \frac{\left(-\frac{1}{k}\right)^{n}}{n !}(-1)^{m} \Lambda^{(m)}(-n)$

9. $\quad \Gamma_{k}\left(c_{1}-z\right) \Gamma_{k}\left(z-c_{2}\right)=\left(\frac{2 \pi}{e}\right)^{2} \delta\left(c_{1}-c_{2}\right), \forall \Lambda(z) \in Z$.

10. $\left\langle F\left[\Gamma_{k}(z)\right], \Lambda(z)\right\rangle=\left\langle\Gamma_{k}(z), F[\Lambda](z)\right\rangle$

11. $\left\langle F\left[\Gamma_{k}(z)\right], F[\Lambda(z)]\right\rangle=2 \pi\left\langle\Gamma_{k}(t), \Lambda(-t)\right\rangle$

12. $\left\langle\overline{\Gamma_{k}(z)}, F[\Lambda(z)]\right\rangle=2 \pi\left\langle\Gamma_{k}(t), \Lambda^{t}(t)\right\rangle$, where $\overline{\Lambda(-t)}=\Lambda^{t}(t)$

13. $\left\langle F\left[\Gamma_{k}(z)\right], \overline{F[\Lambda(z)]}\right\rangle=2 \pi\left\langle\Gamma_{k}(t), \Lambda^{t}(t)\right\rangle$

14. $\left\langle\overline{F\left[\Gamma_{k}(z)\right]}, \overline{F[\Lambda(z)]}\right\rangle=2 \pi\left\langle F\left[\Gamma_{k}(z)\right], F[\Lambda(z)]\right\rangle$

15. $F\left[\Gamma_{k}^{(m)}(z)\right]=\left[(-i t)^{m} \Gamma_{k}(z)\right]$

16. $\Gamma_{k}\left(z+c_{1}\right)=\sum_{n=0}^{\infty} \frac{\left(c_{1}\right)^{n}}{n !} \Gamma_{k}^{(n)}(z)$

where $c_{1}, \Gamma$ and $c_{2}$ are arbitrary real or complex constants.

Proof. The proof of the above results (1-6) is straightforward in view of the characteristics of delta function and the methodology of Theorem 2. Below we prove result 7. By making use of Equation (13), we find:

$$
\left\langle\Gamma_{k}^{m}(z), \Lambda(z)\right\rangle=\sum_{n=0}^{\infty} \frac{\left(-\frac{1}{k}\right)^{n}}{n !}(-1)^{m} \Lambda^{m}(-n),
$$

which is convergent being a product of rapidly decreasing and slowly increasing functions. Next we prove result 8. By using Equation (62), we get

$$
\begin{aligned}
& \left\langle\Gamma_{k}(z+1), \Lambda(z)\right\rangle=\left\langle\Gamma_{k}(z), \Lambda(z-1)\right\rangle, \\
& \Leftrightarrow\left\langle z \Gamma_{k}(z), \Lambda(z)\right\rangle=\left\langle\Gamma_{k}(z), \Lambda(z-1)\right\rangle, \\
& \Leftrightarrow\left\langle\Gamma_{k}(z), z \Lambda(z)\right\rangle=\left\langle\Gamma_{k}(z), \Lambda(z-1)\right\rangle,
\end{aligned}
$$

as required. Next, by making use of Equation (15), we obtain result 9 in the next line $\left\langle\Gamma_{k}\left(\omega_{1}-\right.\right.$ $\left.z) \Gamma_{k}\left(z-\omega_{2}\right), \Lambda(z)\right\rangle=\left(2 \pi \sum_{n=0}^{\infty} \frac{\left(-\frac{1}{k}\right)^{n}}{n !}\right)^{2}\left\langle\delta\left(\omega_{1}-\omega_{2}\right), \Lambda(z)\right\rangle=\left(\frac{2 \pi}{e}\right)^{2}\left\langle\delta\left(\omega_{1}-\omega_{2}\right), \Lambda(z)\right\rangle$. 
The results (10-15) are also straightforward by using the characteristics of delta function for the Fourier transformation. First, we consider proving result 10.

$$
\begin{aligned}
& \left\langle F\left[\Gamma_{k}(z)\right], \Lambda(z)\right\rangle=2 \pi \sum_{n=0}^{\infty} \frac{\left(-\frac{1}{k}\right)^{n}}{n !}\langle F[\delta(z+k n)], \Lambda(z)\rangle, \\
= & 2 \pi \sum_{n=0}^{\infty} \frac{\left(-\frac{1}{k}\right)^{n}}{n !}\langle\delta(z+k n), F[\Lambda(z)]\rangle,=\left\langle\Gamma_{k}(z), F[\Lambda(z)]\right\rangle .
\end{aligned}
$$

Similarly, result 11 is proven in the next line

$$
\left\langle F\left[\Gamma_{k}(z)\right], \Lambda(z)\right\rangle=2 \pi\left\langle F\left[\Gamma_{k}(z)\right], F[\Lambda(s)]\right\rangle=2 \pi\left\langle\Gamma_{k}(t), \Lambda(-t)\right\rangle=2 \pi\left\langle\Gamma_{k}(t), \Lambda^{t}(t)\right\rangle,
$$

and result 12 is as follows

$$
\left\langle\overline{\Gamma_{k}(z)}, F[\Lambda(s)]\right\rangle=2 \pi\left\langle F\left[\Gamma_{k}(z)\right], \overline{F[\Lambda(s)]}\right\rangle=2 \pi\left\langle F\left[\Gamma_{k}(z)\right], \overline{\Lambda(-t)}\right\rangle=2 \pi\left\langle\Gamma_{k}(t), \Lambda^{t}(t)\right\rangle,
$$

where $\Lambda^{t}$ denotes the transpose of test function $\Lambda$. The above result 13 follows $\left\langle F\left[\Gamma_{k}(z)\right], \overline{F[\Lambda(z)]}\right\rangle=$ $2 \pi\left\langle F\left[\Gamma_{k}(z)\right], \overline{\Lambda(-t)}\right\rangle=2 \pi\left\langle\Gamma_{k}(t), \Lambda^{t}(t)\right\rangle$ and result 14 is proven as

$$
\left\langle\overline{F\left[\Gamma_{k}(z)\right]}, \overline{F[\Lambda(s)]}\right\rangle=2 \pi\left\langle F\left[\Gamma_{k}(z)\right], \overline{\overline{F[\Lambda(s)}]}\right\rangle=2 \pi\left\langle F\left[\Gamma_{k}(z)\right], F[\Lambda(s)]\right\rangle,
$$

where we have considered the Parseval's identity of Fourier transformation. To prove the next result 15 , we get

$$
\begin{gathered}
\left\langle\Gamma_{k}^{(1)}(z), \Lambda(z)\right\rangle=\left\langle F\left[\Gamma_{k}(z)\right], \Lambda^{(1)}(z)\right\rangle=\left\langle F\left[\Gamma_{k}^{(1)}(z)\right], \Lambda(z)\right\rangle=\left\langle\Gamma_{k}(z), F\left[\Lambda^{(1)}(z)\right]\right\rangle \\
\left\langle F\left[\Gamma_{k}^{(1)}(z)\right], \Lambda(z)\right\rangle=\left\langle\Gamma_{k}(z),(-i t) \Lambda(z) e^{-i z t}\right\rangle \\
\left\langle\Gamma_{k}^{(1)}(z), \Lambda(z)\right\rangle=\left\langle(-i t) F\left[\Gamma_{k}(z)\right],\right. \\
\Lambda(z)\rangle\left\langle F\left[\Gamma_{k}^{(1)}(z)\right], \Lambda(z)\right\rangle=\left\langle(-i t) F\left[\Gamma_{k}(z)\right], \Lambda(z)\right\rangle .
\end{gathered}
$$

and so on, we get

$$
\left\langle F\left[\Gamma_{k}^{(m)}(z)\right], \Lambda(z)\right\rangle=\left\langle(-i t)^{m} F\left[\Gamma_{k}(z)\right], \Lambda(z)\right\rangle .
$$

That is the required result and the last result 16 holds by using the fact stated and proved in ([27], p. 201), "If $\mathrm{f}$ is in ' and $\alpha$ is a complex constant then the shifting of $f$ by the amount $-\alpha$ is given by $f(z+\alpha)=\sum_{n=0}^{\infty} \frac{(\alpha)^{n}}{n !} f^{(n)}(z) . "$. Therefore, we have $\left\langle\Gamma_{k}\left(z+c_{1}\right), \Lambda(z)\right\rangle=\left\langle\Gamma_{k}(z), \Lambda\left(z-c_{1}\right)\right\rangle=$ $\lim _{v \rightarrow \infty}\left\langle\Gamma_{k}(z), \sum_{n=0}^{v} \frac{\left(-c_{1}\right)^{n}}{n !} \Lambda^{(n)}(z)\right\rangle=\lim _{v \rightarrow \infty}\left\langle\sum_{n=0}^{v} \frac{\left(c_{1}\right)^{n}}{n !} \Gamma_{k}^{(n)}(z), \Lambda(z)\right\rangle$, which yields the required result.

Remark 4. Fourier transformation is a continuous linear mapping of $D^{\prime}$ onto $Z^{\prime}$ and the inverse Fourier transformation has the same properties as a mapping of $Z^{\prime}$ onto $D^{\prime}$ ([27], p. 203). Hence, (49) shows that $e^{v x} \exp \left(-\frac{e^{k x}}{k}\right)$ is an element of $D^{\prime}$, similarly by considering Equation (50) and inverse Fourier transform then $F\left\{\Gamma_{k}(z)\right\}$ is also in $D^{\prime}$.

\section{Discussion and Future Directions}

The fusion of generalized functions and standard integral transformations has been established extensively in the theory of partial differential equations. This fusion can do various physical and engineering problems that cannot be cracked by established procedures. During this study, we obtained a new understanding for the $k$-gamma function in relation with delta function such that the definition of these functions is formalized for entire test functions of a specific space symbolized by $Z$. This is substantial for evolving the fundamentals of distributional notions for k-gamma function and 
improving their use to solve different problems. For example, considering the Riemann zeta function in domain $0<\Re(z)<1$, we have:

$$
\left\langle\Gamma_{k}(z), \zeta(z)\right\rangle=2 \pi \sum_{n=0}^{\infty} \frac{\left(-\frac{1}{k}\right)^{n}}{n !}\langle\delta(z+n k), \zeta(z)\rangle=2 \pi \sum_{n=0}^{\infty} \frac{\left(-\frac{1}{k}\right)^{n}}{n !} \zeta(-n k),
$$

And for $k=2$, we have an integral of Gaussian function:

$$
\left\langle\Gamma_{2}(z), \zeta(z)\right\rangle=2 \pi \sum_{n=0}^{\infty} \frac{\left(-\frac{1}{2}\right)^{n}}{n !}\langle\delta(z+2 n), \zeta(z)\rangle=2 \pi \sum_{n=0}^{\infty} \frac{\left(-\frac{1}{k}\right)^{n}}{n !} \zeta(-2 n)=0,
$$

And for $k=1$, we develop the following elegant result:

$$
\langle\Gamma(z), \zeta(z)\rangle=2 \pi \sum_{n=0}^{\infty} \frac{\left(-\frac{1}{k}\right)^{n}}{n !} \zeta(-n)=\frac{2 \pi}{e-1}-2 \pi .
$$

$k$-gamma functions precisely specify the basic gamma functions. This component is precise for subsequent new identities for gamma functions. The k-gamma functions repeatedly arose for a variety of perspectives, for instance, perturbative computation of Feynman integrals, the combinatory of creation and annihilation operators. Their new representation explored in this research will enhance the impending uses of these representations in physics, statistics and associated sciences. The procedure to achieve the results by means of new representation discovers an essential straightforwardness that is always needed. These are quite a few illustrations. It is anticipated that the methodology established in this exploration will be substantial for additional investigations of k-gamma functions in future research.

Acknowledgments: The author would like to thank Deanship of Scientific Research at Majmaah University for providing excellent research facilities. The author is also thankful to the anonymous reviewers for their useful comments. They really improved the quality of this manuscript.

Conflicts of Interest: The authors declare no conflict of interest.

\section{References}

1. Diaz, R.; Pariguan, E. On hypergeometric functions and Pochhammer k-symbol. Divulg. Math. 2007, 15, 179-192.

2. Diaz, R.; Pariguan, E. Feynman-Jackson integrals. J. Nonlinear Math. Phys. 2006, 13, 365-376. [CrossRef]

3. Karwowski, J.; Witek, A.H. Biconfluent Heun equation in quantum chemistry: Harmonium and related systems. Theor. Chem. Acc. 2014, 133, 1494. [CrossRef]

4. Lackner, M.; Lackner, M. On the likelihood of single-peaked preferences. Soc. Choice Welf. 2017, 48, 717-745. [CrossRef] [PubMed]

5. Kilbas, A.A.; Srivastava, H.M.; Trujillo, J.J. Theory and Applications of Fractional Differential Equations; North-Holland Mathematical Studies; Elsevier (North-Holland) Science Publishers: Amsterdam, The Netherlands; London, UK; New York, NY, USA, 2006; Volume 204.

6. Agarwal, P.; Chand, M.; Baleanu, D.; O'Regan, D.; Shilpi, J. On the solutions of certain fractional kinetic equations involving k-Mittag-Leffler function. Adv. Differ. Equ. 2018, 1, 249. [CrossRef]

7. Set, E.; Tomar, M.; Sarikaya, M.Z. On generalized Grüss type inequalities for k-fractional integrals. Appl. Math. Comput. 2015, 269, 29-34. [CrossRef]

8. Diaz, R.; Teruel, C. q, k-generalized gamma and beta functions. J. Nonlinear Math. Phys. 2005, 12, 118-134. [CrossRef]

9. Diaz, R.; Pariguan, E. On the Gaussian q-distribution. J. Math. Anal. Appl. 2015, 358, 1-9. [CrossRef] 
10. Fernández-Navarro, F.; Hervás-Martínez, C.; Gutiérrez, P.A.; Peña-Barragán, J.M.; López-Granados, F. Parameter estimation of q-gaussian radial basis functions neural networks with a hybrid algorithm for binary classification. Neurocomputing 2012, 75, 123-134.

11. Rehman, A.; Mubeen, S. Some inequalities involving k-gamma and k-beta functions with applications-II. J. Inequal. Appl. 2014, 2014, 445. [CrossRef]

12. Díaz, R.; Ortiz, C.; Pariguan, E. On the k-gamma q-distribution. Open Math. 2010, 8, 448-458.

13. Agarwal, P.; Chand, M.; Choi, J.; Singh, G. Certain fractional integrals and image formulas of generalized k-bessel function. Commun. Korean Math. Soc. 2018, 33, 423-436.

14. Nisar, K.S.; Mondal, S.R.; Choi, J. Certain inequalities involving the k-Struve function. J. Inequal. Appl. 2017, 1, 71. [CrossRef] [PubMed]

15. Chaudhry, M.A.; Qadir, A. Fourier transform and distributional representation of Gamma function leading to some new identities. Int. J. Math. Math. Sci. 2004, 37, 2091-2096. [CrossRef]

16. Tassaddiq, A.; Qadir, A. Fourier transform and distributional representation of the generalized gamma function with some applications. Appl. Math. Comput. 2011, 218, 1084-1088. [CrossRef]

17. Tassaddiq, A.; Qadir, A. Fourier transform representation of the extended Fermi-Dirac and Bose-Einstein functions with applications to the family of the zeta and related functions. Integral Transforms Spec. Funct. 2018, 22, 453-466. [CrossRef]

18. Tassaddiq, A. Some Representations of the Extended Fermi-Dirac and BoseEinstein Functions with Applications. Ph.D. Dissertation, National University of Sciences and Technology Islamabad, Islamabad, Pakistan, 2011.

19. Al-Lail, M.H.; Qadir, A. Fourier transform representation of the generalized hypergeometric functions with applications to the confluent and gauss hypergeometric functions. Appl. Math. Comput. 2015, 263, 392-397. [CrossRef]

20. Tassaddiq, A. A New Representation of the Extended Fermi-Dirac and Bose-Einstein Functions. Int. J. Math. Anal. Appl. 2017, 5, 435-446.

21. Tassaddiq, A.; Safdar, R.; Kanwal, T. A distributional representation of gamma function with generalized complex domain. Adv. Pure Math. 2017, 7, 441-449. [CrossRef]

22. Tassaddiq, A. A new representation of the Srivastava $\lambda$-generalized Hurwitz-Lerch zeta functions. Symmetry 2018, 10, 733. [CrossRef]

23. Prudnikov, A.P.; Brychkov, Y.A.; Marichev, O.I. Integrals and Series: Elementary Functions; Gordon \& Breach Science Publishers: New York, NY, USA, 1992; Volume 1-V.

24. Olver, F.W.J.; Lozier, D.W.; Boisvert, R.F.; Clark, C.W. (Eds.) NIST Handbook of Mathematical Functions; [With 1 CD-ROM (Windows, Macintosh and UNIX)]; U.S. Department of Commerce, National Institute of Standards and Technology: Washington, DC, USA, 2010.

25. Chaudhry, M.A.; Zubair, S.M. On a Class of Incomplete Gamma Functions with Applications; Chapman and Hall (CRC Press Company): Boca Raton, FL, USA; London, UK; New York, NY, USA; Washington, DC, USA, 2001.

26. Gel'fand, I.M.; Shilov, G.E. Generalized Functions: Properties and Operations; Academic Press: New York, NY, USA, 1969; Volume 1-V.

27. Zamanian, A.H. Distribution Theory and Transform Analysis; Dover Publications: New York, NY, USA, 1987.

28. Richards, I.; Youn, H. Theory of Distributions: A Non Technical Introduction; Cambridge University Press: Cambridge, MA, USA; London, UK; New York, NY, USA, 2007.

29. Hoskins, R.F.; Pinto, J.S. Theories of Generalised Functions: Distributions, Ultradistributions and Other Generalised Functions, 2nd ed.; Elsevier: Amsterdam, The Netherlands, 2005.

30. Lebedeve, N.N. Special Functions and Their Applications; Prentice Hall: Englewood Cliffs, NJ, USA, 1965.

(C) 2019 by the author. Licensee MDPI, Basel, Switzerland. This article is an open access article distributed under the terms and conditions of the Creative Commons Attribution (CC BY) license (http://creativecommons.org/licenses/by/4.0/). 